\title{
Designing an efficient rectifying cut-wire metasurface for electromagnetic energy harvesting
}

\author{
Gabin T. Oumbé Tékam, ${ }^{1,2}$ Vincent Ginis, ${ }^{1}$ Jan Danckaert, ${ }^{1}$ and Philippe Tassin ${ }^{1,3}$ \\ ${ }_{1}^{1}$ Applied Physics Research Group (APHY), Vrije Universiteit Brussel, Pleinlaan 2, B-1050 Brussel, Belgium \\ ${ }^{2}$ Laboratory of Modelling and Simulation in Engineering, Biomimetics and Prototypes, Faculty of Science, \\ University of Yaounde I, P.O. Box 812, Yaounde, Cameroon \\ ${ }^{3}$ Department of Physics, Chalmers University, SE-412 96 Göteborg, Sweden
}

(Received 14 November 2016; accepted 4 February 2017; published online 21 February 2017)

\begin{abstract}
Electromagnetic energy harvesting, i.e., capturing energy from ambient microwave signals, may become an essential part in extending the battery lifetime of wearable devices. Here, we present a design of a microwave energy harvester based on a cut-wire metasurface with an integrated PN junction diode. The cut wire with a quasistatic electric-dipole moment is designed to have a resonance at $6.75 \mathrm{GHz}$, leading to a substantial cross-section for absorption. The external microwaves create a unidirectional current through the rectifying action of the integrated diode. Using an electrical-circuit model, we design the operating frequency and the resistive load of the cut wire. Subsequently, by optimizing our design using full-wave numerical simulations, we obtain an energy harvesting efficiency of $50 \%$ for incident power densities in agreement with the typical power density of WiFi signals. Finally, we study the effect of connecting adjacent unit cells of the metasurface in parallel by a thin highly inductive wire and we demonstrate that this allows for the collection of current from all individual cells, while the microwave resonance of the unit cell is not significantly altered, thus solving the wiring problem that arises in many nonlinear metamaterials.
\end{abstract} Published by AIP Publishing. [http://dx.doi.org/10.1063/1.4976804]

Energy harvesting is the emerging field of research focused on the recovering of ambient energy that is otherwise lost to the environment, e.g., vibrational energy, thermal energy, solar energy, and even the microwave energy typically used in wireless communication systems. Vibrational energy is harvested using electromechanical systems. These systems are essentially made of two parts: a mechanical part that collects and enhances the ambient vibrations and an electrical part that converts the vibrations into electrical energy via a transducer. ${ }^{1-7}$ Thermoelectric generators are used to harvest thermal energy. Such a component consists of a closed loop formed by two different metals joined in two places, where a temperature gradient between the junctions creates a voltage difference across the metals. ${ }^{8,9}$ The most popular form of energy harvesting is the collection of sunlight energy and its conversion into electrical energy using solar cells. ${ }^{10,11}$ Microwave electromagnetic energy, finally, is the ambient energy source that has grown substantially in the last decade, as a result of the inherent inefficiency of electromagnetic energy transfer in wireless communication broadcasting systems. While this energy is normally lost, it could be used instead to power small electronic devices such as wearables.

A first successful attempt towards the conversion of microwave electromagnetic energy to DC electrical power was made using a single rectenna harvester. ${ }^{12-15}$ More recently, metamaterials ${ }^{16-24}$ and metasurfaces ${ }^{25-29}$ were proposed for this purpose. ${ }^{30-32}$ Indeed, metamaterials can be designed to resonate at particular frequencies depending on their shape, geometry, size, and orientation, and they provide a powerful platform for electromagnetic wave manipulation. Typically, metamaterials can have a higher density of interacting units than an array of microstrip patch antennas, allowing for higher energy conversion efficiencies. ${ }^{32}$ Furthermore, by designing a metamaterial energy harvester resonating at terahertz frequencies, it is even possible to harvest solar energy, ${ }^{33,34}$ resulting in hybrid solar and electromagnetic energy harvesters. ${ }^{14}$ Ramahi et al. were the first to study electromagnetic energy harvesting with metamaterials. ${ }^{30}$ They designed a metamaterial energy harvester made from splitring resonators (SRRs), operating at $5.8 \mathrm{GHz}$. A resistance was inserted within the gap, thereby directly channeling the RF energy to power a resistive load. To increase the efficiency of this device even further, a stacked array of the same unit cell was developed by Almoneef and Ramahi in Ref. 35 and a ground plane was added in Ref. 36.

To increase the practical usability of these energy harvesters, the microwave energy needs to be converted into DC current. This was first achieved experimentally by Hawkes et al. ${ }^{37}$ by integrating a Greinacher circuit as a rectifier within a split-ring resonator. This electrical circuit has two Schottky diodes in parallel that double the output voltage, allowing for $36.8 \%$ of the incident power to be converted to DC power for a $5 \times 1$ array. A key technical challenge remains how to integrate a rectifier element inside the metamaterial harvester without substantially degrading its efficiency. For this purpose, Duan et al. ${ }^{38}$ used the technique of tunable metamaterial absorbers, consisting of an electric inductive-capacitive (ELC) metamaterial, a dielectric substrate, and a ground plane, and integrated a Schottky diode into the metamaterial unit cell that acts as a rectifier. Despite these efforts, the efficient recovery of microwave energy sources remains a challenge and the development of suitable microwave energy harvesters has become an increasingly important question, particularly at realistic incident microwave power levels. 
In this Letter, we propose a purely planar metasurface energy harvester based on a cut-wire metamaterial unit cell with a PN junction diode embedded in each unit cell, as shown in Fig. 1. There is no DC bias voltage applied to the $\mathrm{PN}$ junction, so a biasing circuit is not required, and we consider a realistic amplitude of the input signal corresponding to the field intensities typically observed for WiFi signals.

To understand how this metasurface allows for efficient energy harvesting, it can be instructive to think about the interaction of a simple diode without the surrounding resonator. When an electromagnetic wave with an amplitude of approximately $20 \mathrm{~V} / \mathrm{m}$ illuminates such a free-standing diode, the voltage drop over the contacts of the diode is much too small ( $\ll 100 \mathrm{mv})$ to overcome the threshold voltage of the diode. As a result, barely any current will flow through the element. However, the situation changes significantly by adding a resonator, allowing for large electric field enhancement and larger voltage differences across the diode. Metasurface cut-wire elements are ideal candidates for this
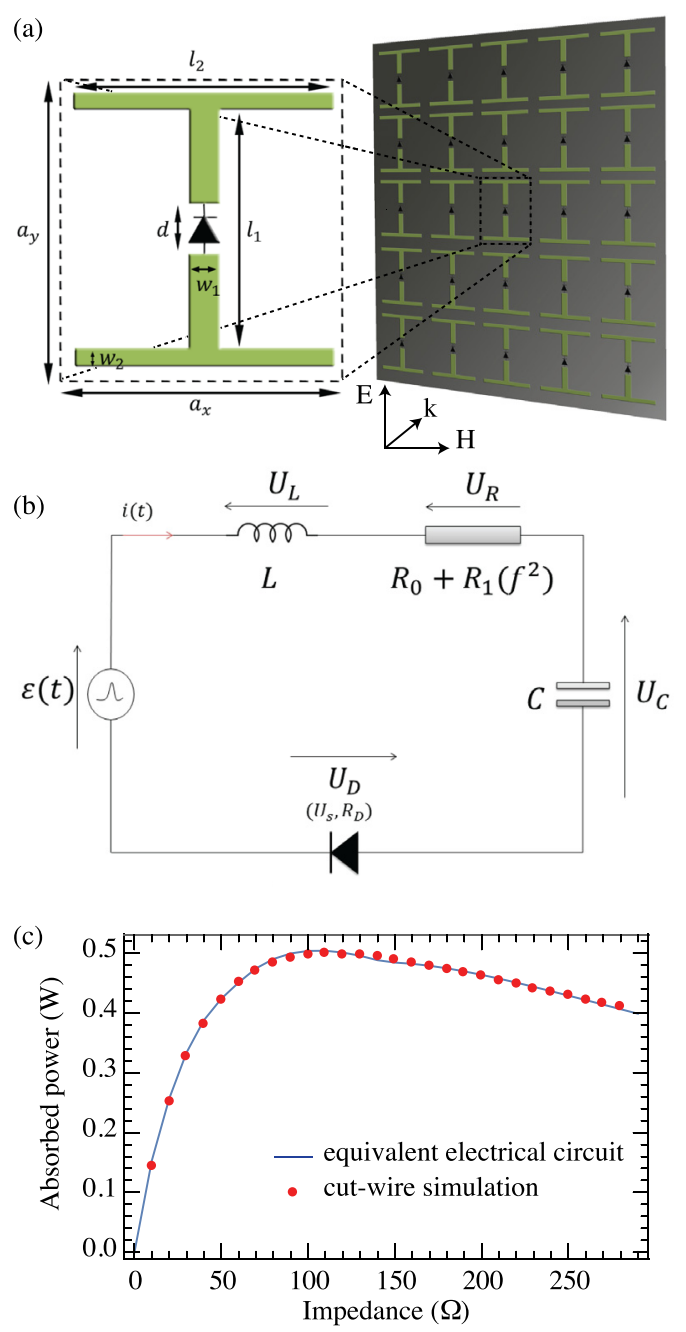

FIG. 1. (a) The metasurface energy harvester composed of cut-wire cells with an integrated diode. (b) The equivalent electrical circuit of a single unit cell. (c) The absorbed power inside the cut wire (without diode) as a function of the impedance $\left(Z_{P}\right)$ of a resistance inserted in the middle of the unit cell. The size of the unit cell is: $l_{1}=23.6 \mathrm{~mm}, l_{2}=22.8 \mathrm{~mm}, w_{1}=3 \mathrm{~mm}$, $w_{2}=1.6 \mathrm{~mm}, d=2.95 \mathrm{~mm}$, and $a_{x}=a_{y}=30 \mathrm{~mm}$. The electrical parameters are: $L=43.28 \mathrm{nH}, C=0.07 \mathrm{pF}, R_{0}=1.62 \mathrm{~m} \Omega$, and $R_{1}=1.35 \times$ $10^{-22} \times f^{2} \Omega / \mathrm{Hz}^{2}$. The input voltage amplitude $\varepsilon_{0}$ equals $13 \mathrm{~V}$. purpose, because they have a large electric dipole moment for improved collection of external radiation and they can be closely packed with a distance between two cut-wire unit cells smaller than a fraction of the wavelength, in order to consider the device as an effective metasurface and to avoid diffraction effects. Therefore, the coupling between adjacent unit cells cannot be neglected.

Indeed, when an electromagnetic plane wave propagates through such a metasurface unit cell, the electric dipole resonance of the cut wires is excited and an electric current is induced in the structure. The dynamics of this current is very similar to that of an RLC circuit with the coupling effects included in the electric parameters (see Fig. 1(b)). The inductance $L$ of the resonator can be engineered by altering the length and the width of the cut wire and the capacitance $C$ is predominantly determined by the end caps of the structure. The resistance $R$ has two contributions here: the ohmic loss $R_{0}$, related to the dissipative loss in the metal, and the radiation loss $R_{1}$, related to the reradiation of electromagnetic waves by the cut wire. The radiation resistance is proportional to the square of the input wave frequency $(f)^{39,40}$

Although the electric field is enhanced considerably by the cut-wire metamaterial and absorption is enhanced, the captured field usually reradiates rapidly due to the electricdipole coupling to the free space modes in the absence of a nonlinear element. Therefore, we insert a PN junction diode within each unit cell of our metasurface. ${ }^{41}$ To this end, a narrow gap is created in the middle of the cut-wire as shown in Fig. 1(a), where the diode is inserted. The parameters of the diode are determined such that the power transfer into the unit cell is optimized. This is done in a two-step procedure (see the supplementary material). First, we insert a resistance load as a lumped element in the narrow gap, where the diode is going to be placed. By sweeping the impedance $\left(Z_{P}\right)$ of the lumped element in a full-wave numerical simulation, we find that the maximum power transfer is obtained at $Z_{P} \approx 100 \Omega$, as shown in Fig. 1(c). The same results are retrieved from the equivalent electrical circuit using a PSPICE electrical circuit simulator. Second, the lumped element is replaced by a diode, whose characteristic resistance $R_{D}$ in series with the wire resistance equals the impedance of the lumped element. The complete unit cell now is equivalent to an $R L C$ circuit in series with a diode, as shown in Fig. 1(b).

To verify our intuitive model and to gain insight into the role of the diode implemented in the metasurface, we perform full-wave numerical simulations using a finite-element electromagnetic solver. We illuminated the metasurface by an incident field generated on a distant surface by a surface current with a Gaussian time profile given by

$$
J_{s}=J_{s 0} \sin (2 \pi f t) e^{-\left(\frac{t-t_{0}}{\Delta t}\right)^{2}},
$$

where $J_{s 0}$ is the maximal amplitude of the surface current density and the relationship between $J_{s}$ and the incident electric field $E_{\text {in }}$ is $J_{s}=\frac{2 E_{\text {in }}}{\zeta}$ (see the supplementary material). The amplitude of the incident electric field is $20.5 \mathrm{~V} / \mathrm{m}$ corresponding to an incident power density of $1.13 \mathrm{~W} / \mathrm{m}^{2}$, which is approximately the amplitude of microwave electric fields in wireless communication systems. The characteristic 
current-voltage relation of the diode in series with the resistance is modeled by the following relation:

$$
I_{D}=\left\{\begin{array}{lll}
0 & \text { if } & U_{D} \leq U_{s} \\
\frac{U_{D}-U_{s}}{R_{D}} & \text { if } & U_{D}>U_{s}
\end{array}\right.
$$

where $U_{s}$ is the threshold voltage of the diode. We assumed mirror symmetric boundary conditions compatible with the incident electric field polarization in order to model a periodic array of the cut-wire metamaterial energy harvester. ${ }^{42}$ Doing so, we include all potential coupling effects with neighbouring unit cells and make a very realistic simulation of the performance. To visualize the temporal evolution of the electric field across the diode, we solve for the electric fields using a time-domain analysis. A typical result of this analysis is shown in Fig. 2. This figure clearly visualizes how the incident electromagnetic radiation is converted into DC electric power for a frequency of $f=3 \mathrm{GHz}$ and a threshold voltage of $0.3 \mathrm{~V}$ (typical for a germanium diode).

Instead of oscillating and reradiating, the voltage across the cap of the thin wire will stabilize to a constant value. From the saturation voltage, we can estimate the energy harvested by the nonlinear metasurface. Indeed, this voltage is generated through the accumulation of charges between two ends of the cut-wire metamaterial, resulting from the unidirectional flow through the diode. From Fig. 2, we can observe a harvested voltage of around $0.13 \mathrm{~V}$ for a power density of $1.13 \mathrm{~W} / \mathrm{m}^{2}$ at a frequency of $3 \mathrm{GHz}$. We can obtain the same results from the equivalent electrical circuit by considering an input voltage with the same form as Eq. (1) at the same frequency and taking the characteristic current-voltage relation $I_{D}=I_{S}\left(e^{\left(\frac{U_{D}-U_{s}}{n V_{T}}\right)}-1\right)$. This result demonstrates that the cut-wire metasurface with an integrated diode is able to harvest electromagnetic energy and to transform a microwave field pulse into a DC voltage without

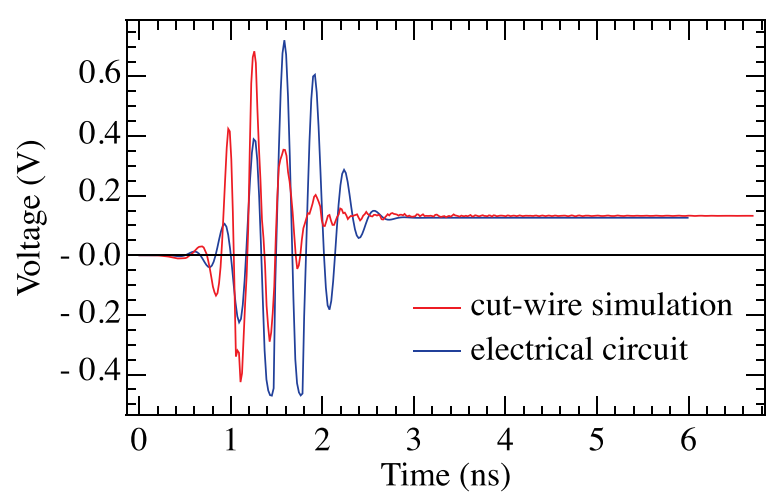

FIG. 2. The time evolution of the voltage across the diode for the computer simulation of the cut-wire metamaterial energy harvester and its equivalent electrical circuit for a power density of $1.13 \mathrm{~W} / \mathrm{m}^{2}$ at a frequency of $3 \mathrm{GHz}$. The parameters of the diode in PSPICE are $I_{s}=10^{-12} \mathrm{~A}, U_{s}=0.3 \mathrm{~V}, n=1$, extra internal capacitance of the diode $C_{s}=10^{-6} \mathrm{pF}$, and the input voltage amplitude $\varepsilon_{0}=0.67 \mathrm{~V}$. The discrepancy in the transient region is probably due to a subtle limitation of the electric-circuit model with a nonlinear diode. In practice, there are charge carriers sitting not only at the end caps of the cut wire, but also along the entire edge. In a linear circuit, this can all be taken together in a single capacitance, but with inclusion of the nonlinear diode, the local energy distribution influences the transient response. Nevertheless, the steady-state solution is not affected. the need for a DC bias voltage to trigger the functionality of the rectifier.

We have repeated the previous time-domain simulation for many incident frequencies and for two different kinds of diodes, allowing for an investigation of the bandwidth and of the effect of the threshold voltage on the efficiency of the metasurface energy harvester. We considered silicon diodes with threshold voltage $U_{s}=0.7 \mathrm{~V}$ and germanium diodes with $U_{s}=0.3 \mathrm{~V}$. The efficiency $\eta$ is calculated as $\eta=W_{\text {out }} /$ $W_{\text {in }}$, where we determine the output energy $W_{\text {out }}$ by evaluating the saturation voltage $\left(U_{c}\right)$ and calculating the stored energy on the capacitor by $W_{\text {out }}=C U_{c}^{2} / 2$. The capacitance of the cut-wire $C$ is retrieved numerically. The incident power $W_{i n}$ is found by integrating the Poynting vector over the unit cell area $A$ (see the supplementary material)

$$
W_{i n}=\frac{E_{\text {in }}^{2} \Delta t}{4 \zeta} A \sqrt{\frac{\pi}{2}}\left(1-e^{-2(\pi f \Delta t)^{2}}\right) .
$$

The resulting energy conversion efficiency is shown in Fig. 3 as a function of the center frequency of the incident field. The thin-wire resonator with an integrated diode clearly shows a resonant response close to $6.75 \mathrm{GHz}$. The addition of the diode shifted this resonance frequency compared to the traditional thin wire (see the supplementary material), due to the extra internal capacitance of the diode (the resonance frequency can still be chosen at will by modifying the geometric capacitance of the cut-wire). It is remarkable to find that on resonance up to $50 \%$ of the incident energy can be collected and transformed into DC energy. Note that a 50\% collection efficiency is the maximum that can be expected from an electric-dipole-only metasurface-for higher efficiency a magnetic dipole is needed. We also observe that the use of a germanium diode $\left(U_{s}=0.3 \mathrm{~V}\right)$ increases the conversion efficiency of the energy harvester fivefold (from $12.5 \%$ to $50 \%$ at $6.75 \mathrm{GHz})$ compared to when a silicon diode is used. Along the same line, we notice that the bandwidth is larger using a germanium diode compared to a silicon diode. Finally, we also find that the threshold voltage does not affect the resonance frequency. These results are perfectly in agreement with the intuitive model described in the first part of this letter. Indeed, a smaller threshold voltage allows for larger currents to flow for a given voltage difference across the diode, hence increasing the energy conversion. At the same time, it allows

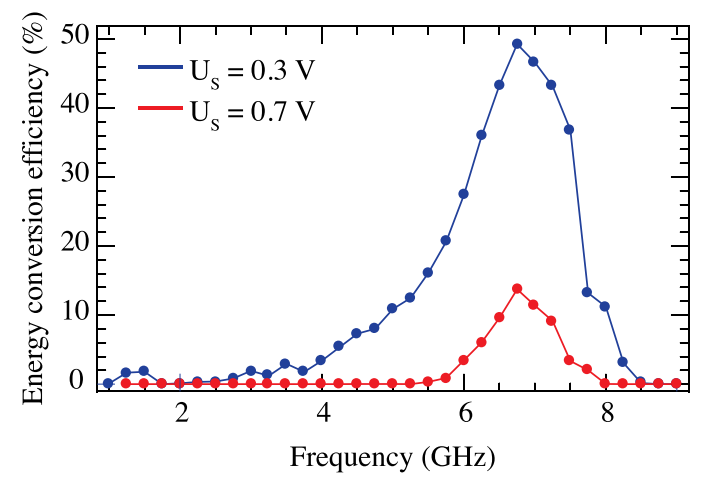

FIG. 3. Energy conversion efficiency of the harvesting metasurface for different values of the threshold voltage. The metasurface with a Germanium diode reaches an energy conversion efficiency of $50 \%$ at resonance. 
for the energy to be harvested at frequencies further away from resonance, explaining the broadening of the bandwidth.

It can be expected that the efficiency changes for varying incident angles. We have performed additional simulations to investigate this effect (see the supplementary material). For waves incident under an angle $\theta$, the impedance of the surface is given by $Z /(\cos \theta)$, where $Z$ is the impedance for perpendicular incidence. The maximum efficiency is obtained for normally incident waves, because the structure was optimized (load matching) for normal incidence. From Fig. S6 (supplementary material), it is also clear that the performance remains high for a broad range of incident angles. In practice, the radiation that we want to harvest is uniformly distributed over all angles. Only the energy in a small angle range will not be accepted efficiently by our harvester, but since this is only a small band it will not substantially affect the efficiency of our device.

In the final part of this Letter, we investigate how the DC energy can be collected from a large array of cut-wire elements. This is an important problem as the harvesting of the DC current requires a conducting path throughout the entire metasurface, possibly adversely affecting the efficiency of the individual cut-wire elements. For this purpose, we add thin metallic wires with high inductance between the end caps of adjacent unit cells [see Fig. 4(a)]. The high inductance of the thin wire inhibits the flow of microwave current between the unit cells. This is confirmed by the results shown in Fig. 4(b), where we compare the capacitor voltage for two different cases: (1) the structure discussed above with disjoint cut-wire elements and (2) the same structure but with the end caps of the cut wires connected by large-inductance conducting lines. We find that the capacitor voltage is almost the same in both cases, with only a very minor decrease in the saturation voltage due to a small shift in resonance frequency originating from the high-inductance lines. This demonstrates that DC electrical energy can be

(a)

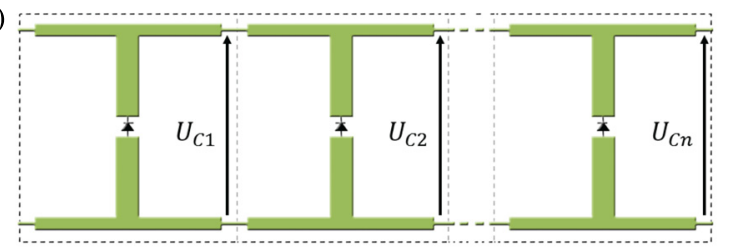

(b)

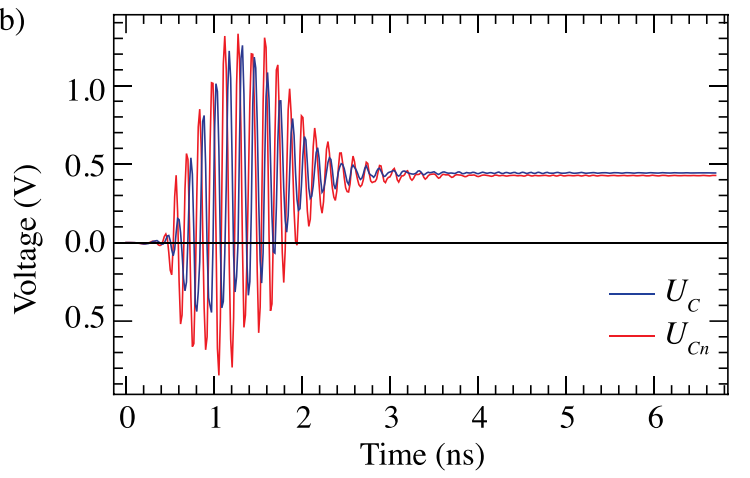

FIG. 4. (a) Connection of the cells to channel the energy harvested. (b) Time evolution of the voltage across an unconnected single cell $\left(U_{C}\right)$ and time evolution of the voltage across a connected cell $\left(U_{C n}\right)$ for a threshold voltage of the diode $U_{s}=0.3 \mathrm{~V}$ at the frequency $f=6.75 \mathrm{GHz}$. easily collected in a large array of the metasurface elements proposed in this Letter.

In summary, we have shown that energy can be harvested from ambient microwave radiation using a cut-wire metasurface, in which an electric dipole resonance is used for electric field enhancement and an integrated diode for rectification of the induced current. Using a combination of circuit models and accurate full-wave simulations, we have optimized our harvesting metasurface to achieve conversion efficiencies close to $50 \%$, the theoretical maximum for a metasurface without a magnetic dipole response. It was also shown that germanium diodes are required for their lower threshold voltage in order to obtain high efficiencies for the typical electric field intensities used in mobile communication networks. An additional advantage of germanium diodes is that they lead to a high bandwidth. Finally, the coupling between adjacent unit cells in the metasurface using thin metallic wires with high kinetic inductance provides a robust mechanism to collect the harvested energy from all cells with barely any degradation of the efficiency of the microwave energy harvester. We are convinced that the subwavelength thin, planar metasurface studied here will lead to future experimental work. A small discrepancy that can be expected in comparing the simulations with experiments can occur because of a non-uniform illumination of the entire surface. Although the fabrication defects, the choice of the diode, and the truncation to a finite array of the metasurface may slightly affect the efficiency, these deviations are moderate and the broadening due to realistic tolerances is typically smaller than the bandwidth of the device. We envision that our design can be used as sustainable power source to drive small sensors and wearable appliances for a plethora of applications.

See supplementary material for a detailed description of the metasurface design, the calculation of the efficiency at oblique incidence, and details of the simulation methods.

G.T.O.T. acknowledges a scholarship from the Caribu Erasmus Mundus Action 2 programme. V.G. acknowledges a fellowship from the Research Foundation Flanders (FWOVlaanderen). Work at VUB was also supported by the Interuniversity Attraction Poles program of the Belgian Science Policy Office under grant IAP P7-35 photonics@be and the research council of the VUB.

\footnotetext{
${ }^{1}$ A. Erturk and D. J. Inman, Piezoelectric Energy Harvesting, 1st ed. (John Wiley \& Sons, Ltd., 2011).

${ }^{2}$ J. Cao, W. Wang, S. Zhou, D. J. Inman, and J. Lin, Appl. Phys. Lett. 107, 143904 (2015).

${ }^{3}$ G. T. O. Tekam, E. B. T. Tchuisseu, C. A. K. Kwuimy, and P. Woafo, Nonlinear Dyn. 76, 1561 (2014).

${ }^{4}$ A. N. Kadjie and P. Woafo, Theor. Appl. Mech. Lett. 4, 063001 (2014).

${ }^{5}$ G. T. O. Tekam, C. A. K. Kwuimy, and P. Woafo, Chaos 25, 013112 (2015).

${ }^{6}$ C. A. K. Kwuimy and C. Nataraj, Struct. Nonlinear Dyn. Diagn. 4, 97-123 (2015).

${ }^{7}$ P. R. N. Tuwa and P. Woafo, J. Mech. Med. Biol. 16, 1650073 (2016).

${ }^{8}$ N. Hudak and G. Amatucci, J. Appl. Phys. 103, 101301 (2008).

${ }^{9}$ G. S. Nolas, J. Sharp, and H. J. Goldsmid, Thermoelectrics: Basic Principles and New Materials Developments (Springer, New York, 2001).

${ }^{10}$ C. Alippi and C. Galperti, IEEE Trans. Circuits Syst. I: Regular Pap. 55, 1742-1750 (2008).
} 
${ }^{11}$ Y. Li, N. J. Grabham, S. P. Beeby, and M. J. Tudor, Solar Energy 111, 21-29 (2015).

${ }^{12}$ J. A. Hagerty and Z. B. Popovic, IEEE MTT-S Int. Microwave Symp. Dig. 3, 1855-1858 (2001).

${ }^{13}$ N. Zhu, R. W. Ziolkowski, and H. Xin, Appl. Phys. Lett. 99, 114101 (2011).

${ }^{14}$ A. Collado and A. Georgiadis, IEEE Trans. Circuits Syst. I: Regular Pap. 60, 2225-2234 (2013).

${ }^{15}$ V. Palazzi, C. Kalialakis, F. Alimenti, P. Mezzanotte, L. Roselli, A. Collado, and A. Georgiadis, in IEEE Wireless Power Transfer Conference (2016).

${ }^{16}$ J. P. Pendry, A. J. Holden, D. J. Robbins, and W. J. Stewart, "Low frequency plasmons in thin-wire structures," J. Phys.: Condens. Matter 10, 22 (1998).

${ }^{17}$ D. R. Smith, J. B. Pendry, and M. C. K. Wiltshire, Science 305, 5685 (2004).

${ }^{18}$ R. Engheta and R. W. Ziolkowski, Metamaterials: Physics and Engineering Explorations (Wiley-IEEE Press, New York, 2006).

${ }^{19}$ C. M. Soukoulis and M. Wegener, Nat. Photonics 5, 523 (2011).

${ }^{20}$ N. I. Zheludev and Y. S. Kivshar, Nat. Mater. 11, 917 (2012).

${ }^{21}$ Z. Jiangfeng, L. Zhang, G. Tuttle, T. Koschny, and C. M. Soukoulis, Phys. Rev. B 73, 041101 (2006).

${ }^{22}$ Z. Lei, P. Tassin, T. Koschny, C. Kurter, S. M. Anlage, and C. M. Soukoulis, Appl. Phys. Lett. 97, 241904 (2010).

${ }^{23}$ S. Foteinopoulou, G. Kenanakis, N. Katsarakis, I. Tsiapa, M. Kafesaki, E. N. Economou, and C. M. Soukoulis, Appl. Phys. Lett. 91, 214102 (2007).

${ }^{24}$ Z. Li, R. Zhao, T. Koschny, M. Kafesaki, K. B. Alici, E. Colak, H. Caglayan, E. Ozbay, and C. M. Soukoulis, Appl. Phys. Lett. 97, 081901 (2010).

${ }^{25}$ Y. Yao, R. Shankar, M. A. Kats, Y. Song, J. Kong, M. Loncar, and F. Capasso, Nano. Lett. 14, 6526 (2014).
${ }^{26}$ N. Yu and F. Capasso, Nat. Mater. 13, 139 (2014).

${ }^{27}$ A. V. Kildishev, A. Boltasseva, and V. M. Shalaev, Science 339, 6125 (2013).

${ }^{28}$ V. Ginis, P. Tassin, T. Koschny, and C. M. Soukoulis, Appl. Phys. Lett. 108, 031601 (2016).

${ }^{29}$ C. Kurter, P. Tassin, L. Zhang, T. Koschny, A. P. Zhuravel, A. V. Ustinov, S. M. Anlage, and C. M. Soukoulis, Phys. Rev. Lett. 107, 043901 (2011).

${ }^{30}$ O. M. Ramahi, T. S. Almoneef, M. Alshareef, and M. S. Boybay, Appl. Phys. Lett. 101, 173903 (2012).

${ }^{31}$ S. J. Byrnes, R. Blanchard, and F. Capasso, Proc. Natl. Acad. Sci. 111, 3927-3932 (2014).

${ }^{32}$ B. Alavikia, T. S. Almoneef, and O. M. Ramahi, Appl. Phys. Lett. 107, 033902 (2015).

${ }^{33}$ B. Mulla and C. Sabah, Plasmonics 11(5), 1313-1321 (2016).

${ }^{34}$ M. Alshareef and O. M. Ramahi, J. Appl. Phys. 114, 223101 (2013).

${ }^{35}$ T. S. Almoneef and O. M. Ramahi, Prog. Electromagn. Res. 146, 109-115 (2014).

${ }^{36}$ T. S. Almoneef and O. M. Ramahi, Appl. Phys. Lett. 106, 153902 (2015).

${ }^{37}$ A. M. Hawkes, A. R. Katko, and S. A. Cummer, Appl. Phys. Lett. 103, 163901 (2013).

${ }^{38} \mathrm{X}$. Duan, X. Chen, and L. Zhou, "A metamaterial harvester with integrated rectifying functionality," in International Conference on Wireless Information Technology and Systems (ICWITS) and Applied Computational Electromagnetics (ACES), 2016 IEEE/ACES (IEEE, 2016).

${ }^{39}$ J. D. Jackson, Classical Electrodynamics, 3rd ed. (John Wiley \& Sons, Inc., 1999).

${ }^{40} \mathrm{P}$. Tassin, T. Koschny, M. Kafesaki, and C. M. Soukoulis, Nat. Photonics 6, 259-264 (2012).

${ }^{41}$ A. Sanchez, C. F. Davis, Jr., K. C. Liu, and A. Javan, J. Appl. Phys. 49, 5270 (1978).

${ }^{42}$ P. Tassin, T. Koschny, and C. M. Soukoulis, Phys. B 407, 4062-4065 (2012). 